\title{
Pyruvate Kinase Isozyme M2 Measurement
}

National Cancer Institute

\section{Source}

National Cancer Institute. Pyruvate Kinase Isozyme M2 Measurement. NCI Thesaurus. Code C156531.

The determination of the amount of pyruvate kinase isozyme M2 present in a sample. 